\title{
Dual-Lumen Balloon Catheters May Improve Liquid Embolization of Vascular Malformations: An Experimental Study in Swine
}

\author{
J.C. Gentric, J. Raymond, A. Batista, I. Salazkin, G. Gevry, and T.E. Darsaut
}

\begin{abstract}
BACKGROUND AND PURPOSE: Liquid embolic agents are increasingly used to treat vascular malformations. We sought to assess embolization with these agents by using a dual-lumen balloon catheter in an experimental setting.
\end{abstract}

MATERIALS AND METHODS: Eighteen injections of liquid embolic agents were performed in the rete mirabile of swine. We used 3 methods to control liquid embolic agent reflux: 1) dual-lumen balloon-catheter (group A, $n=8$ ); 2) injection of liquid embolic agent after proximal $n$-BCA plug formation through a second microcatheter (group B, $n=4$ ); and 3) standard liquid embolic agent injection (group C, controls, $n=6$ ). The following outcomes were graded by using ordinal scales by angiography, macrophotography, and radiography of retia after euthanasia: 1) angiographic and pathologic extent of liquid embolic agent embolization of the rete, 2) reflux of liquid embolic agents in the parent artery, and 3) density of liquid embolic agents in the proximal rete. Technical complications were also recorded. A successful injection was defined as an embolization that reached the contralateral rete without reflux into proximal external branches. Exact logistic regression analyses were performed to compare groups.

RESULTS: There were significant differences among groups for reflux $(P=.029)$ and liquid embolic agent density in the proximal rete $(P=$ .014), while extension to the contralateral rete did not reach statistical significance $(P=.07)$. Injections differed among groups $(P=.004)$, with dual-lumen balloon-catheter injections more frequently successful compared with control injections $(P=.019)$.

CONCLUSIONS: Dual-lumen balloon catheters allowed better liquid embolic agent injections than standard injections.

ABBREVIATIONS: LEA = liquid embolic agent; PHIL = Precipitating Hydrophobic Injectable Liquid

$\mathbf{E}_{\text {her }}^{\text {h }}$ hylene-vinyl alcohol copolymer liquid embolic agents (LEAs) have changed the management of vascular malformations such as arteriovenous malformations and dural arteriovenous fistulas. ${ }^{1-4}$ LEAs such as Onyx (Covidien, Irvine, California) can be injected through small microcatheters, and on injection, they precipitate out of the dimethyl-sulfoxide solvent and slowly harden after coming in contact with blood. These agents are cohesive rather than adhesive and perhaps better controlled than other

Received September 2, 2014; accepted after revision November 6.

From the Department of Radiology (I.C.G., J.R., A.B.), Centre Hospitalier de l'Université de Montréal, Notre-Dame Hospital, Montreal, Quebec, Canada; Laboratory of Interventional Neuroradiology (J.R., I.S., G.G.), Centre Hospitalier de l'Université de Montréal, Notre-Dame Hospital Research Centre, Montreal, Quebec, Canada; Division of Neurosurgery (T.E.D.), Department of Surgery, University of Alberta Hospital, Mackenzie Health Sciences Centre, Edmonton, Alberta, Canada; and Equipe d'Accueil 3878 Groupe d'Etude de la Thrombose en Bretagne Occidentale (J.C.G.), Université de Bretagne Occidentale, Brest, France.

This work was supported by MicroVention, which also supplied catheters and the PHIL prototype liquid embolic. Covidien supplied Onyx and microcatheters.

Please address correspondence to Tim Darsaut, MD, University of Alberta Hospital, 8440 112th St, WMC 2C3.83, Edmonton, Alberta, Canada T6G 2B7; e-mail:

tdarsaut@ualberta.ca

http://dx.doi.org/10.3174/ajnr.A4211 agents such as $n$-BCA. In many cases, these features (of LEAs such as Onyx/Phil) may permit longer injections and possibly more complete embolizations than other LEAs. ${ }^{1}$

The standard method used to control injections is to slowly inject the LEA, allowing some reflux for a short plug proximal to the catheter tip to form and harden, and to wait for the LEA to preferentially move in an antegrade direction deep into the nidus of the malformation. One factor potentially limiting the efficacy of the injection is excessive reflux of LEA back along the catheter, which can enter and occlude unwanted vessels. ${ }^{5}$ Additionally, if a segment of microcatheter that is too long is left in contact with the LEA for too long, the proximal plug may entrap the catheter tip in place. Removal of entrapped catheters has led to intracranial hemorrhages, leading to an FDA warning regarding this aspect of treatment with Onyx. ${ }^{6}$

Several potential solutions to these problems have been proposed. One option is to use 2 microcatheters together: One microcatheter with a detachable tip is used for the injection of the LEA, while the other microcatheter is used to deliver $n$-BCA quickly, intentionally gluing the detachable portion of the first 

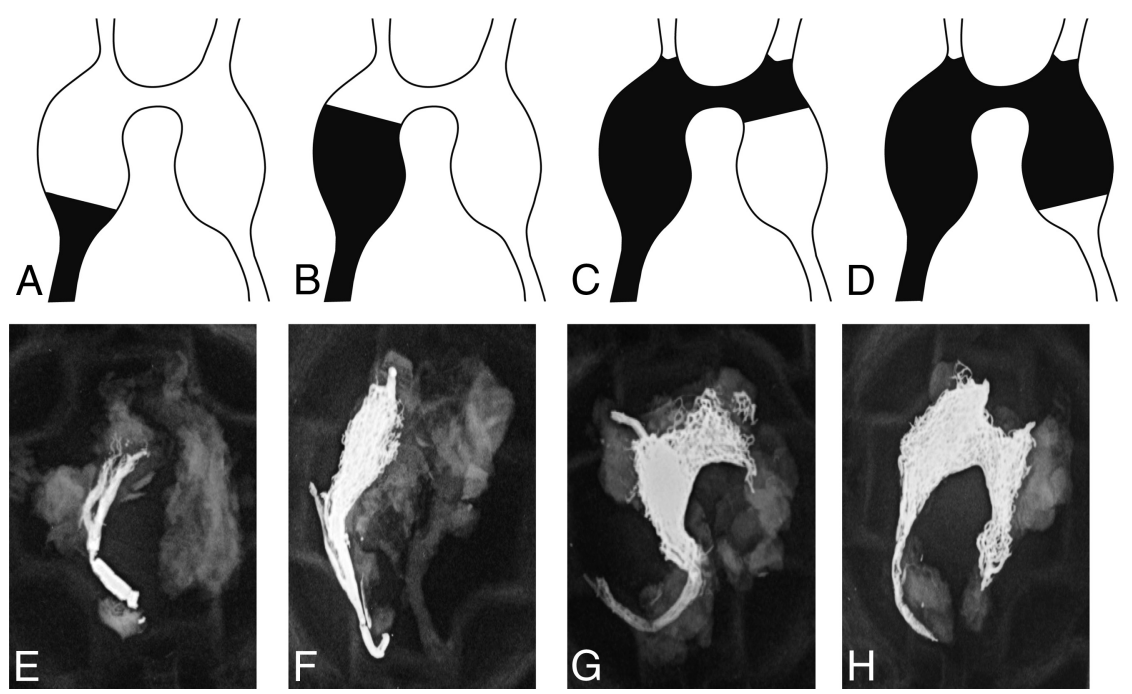

FIG 1. Schematics and radiographs demonstrating the different grades of extent of LEA infiltration through the porcine rete. $A$ and $E$, Grade I, $<50 \%$ infiltration of the ipsilateral rete. $B$ and $F$, Grade II, $>50 \%$ but $<100 \%$ infiltration of the ipsilateral rete. $C$ and $G$, Grade III, complete infiltration through the ipsilateral rete with $<50 \%$ of the contralateral rete. $D$ and $H$, Grade IV, complete infiltration through the ipsilateral and $>50 \%$ of the contralateral rete. proximally and $2.1 \mathrm{~F}$ in its distal segment, with a working lumen inner diameter of 0.0165 inches. The balloon catheter is compatible with up to 0.014 inch guidewires.

Onyx 18 (Covidien) was the LEA used for 11 injections; a prototype LEA (Precipitating Hydrophobic Injectable Liquid [PHIL]; MicroVention) was used for 7 injections. This liquid embolic agent, PHIL, is a 2-component system. The first component is the flushing solution, which is an organic solvent, dimethyl-sulfoxide, and the second component is the liquid embolic agent, a copolymer that is dissolved in dimethyl-sulfoxide.

All injections were performed in random order, either by 2 interventionists (T.E.D. and J.C.G., 5 years of experience each) or by the senior author (J.R., 29 years of experience). All injections were catheter, forming the proximal plug that will prevent reflux of LEA, and ensuring antegrade embolization of the nidus. ${ }^{7-9}$ Another option is to use a dimethyl-sulfoxide-compatible dual-lumen balloon catheter, in which 1 lumen is used to inflate a balloon and prevent reflux, while the second lumen delivers the embolic agent (Scepter; MicroVention, Tustin, California). 5,10

Animal models may be useful to inform clinical applications of embolic agents. ${ }^{11-14}$ We sought to explore whether using a duallumen balloon catheter could improve embolization of the swine rete (a model often used for experimental AVM embolization $)^{12,15,16}$ and prevent excessive reflux compared with more traditional techniques. We hypothesized that the use of the balloon would promote more complete nidus filling with less reflux and fewer complications than other methods.

\section{MATERIALS AND METHODS \\ Embolization}

Protocols for animal experimentation were approved by the Institutional Animal Care Committee in accordance with guidelines of the Canadian Council on Animal Care. All procedures were performed in 20- to 25-kg Yorkshire pigs under general anesthesia. Conventional angiography was performed via femoral puncture. Using a coaxial approach, we navigated microcatheters to the porcine ascending pharyngeal artery, which supplies the rete mirabile. Animals were randomly allocated to be embolized by using 1 of 3 treatment options: 1 ) Group A animals were embolized by using a dual-lumen balloon catheter $(n=8)$; 2) group $\mathrm{B}$ animals were embolized by using a 2-microcatheter technique (1 detachable-tip microcatheter was used for LEA injection [Apollo; Covidien]; the other [Marathon; Covidien] was used for $n$-BCA proximal plug formation $[n=4]$ ); and group $\mathrm{C}$ were controls using a single-microcatheter technique (the balloon microcatheter, keeping the balloon deflated) $(n=6)$.

Scepter is a dimethyl-sulfoxide- and Onyx-compatible double-lumen balloon catheter. The profile of the Scepter is $2.8 \mathrm{~F}$ observed by 2 interventionists. Randomization was performed by flipping a coin for allocation to an experimental group or control and by trying to balance Onyx and prototype LEA injections (1:1) for groups A and C. Thus, the number of injections with Onyx versus PHIL was 4:4 for group A and 3:3 for group C. The number of injections requiring balloon catheters (groups A and C) and LEA and the total number of animals were limited by the availability of material and the total budget for these experiments. Due to these constraints, the resulting final number of animals per group was unequal.

After slowly flushing the microcatheter dead space with dimethyl-sulfoxide and the dimethyl-sulfoxide with the LEA for 2 minutes, we injected the LEA slowly by using fluoroscopic guidance, attempting to reach the contralateral rete, until reflux out of the ascending pharyngeal artery occurred or until the rete was completely filled. Injections were also stopped when the embolic material reached the intracranial circulation. After control angiography, animals were euthanized and the rete mirabile was harvested and immersed in $10 \%$ formalin for macroscopic photography and radiographic and pathologic analyses.

Macroscopic photographs and radiographs of the embolized and formalin-fixed specimens were used to score the extent of LEA infiltration within the rete by using a 4-point scale. Grades were adjudicated by 2 readers (J.R. and J.C.G.) as follows: grade I, $<50 \%$ of the ipsilateral rete; grade II, $>50 \%$ but $<100 \%$ of the ipsilateral rete; grade III, complete infiltration through the ipsilateral rete reaching $<50 \%$ of the contralateral rete; and grade IV, complete infiltration of the ipsilateral and $>50 \%$ of the contralateral rete (Figs 1 and 2 ). We studied the reliability of the grading system: Three authors (J.R., T.E.D., and J.C.G.) blindly and independently scored a random sample of 10 rete radiographs from these experiments to provide a measure of interobserver variability.

Reflux was defined as any retrograde filling of the ascending pharyngeal artery proximal to the catheter tip and was graded by 

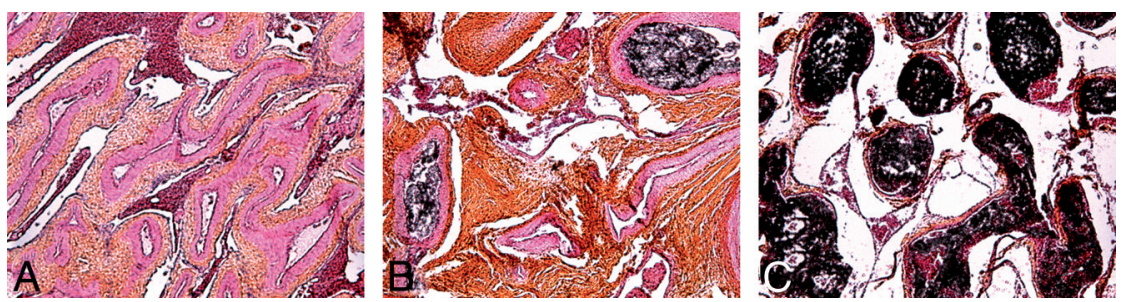

FIG 2. Microscopic pathology of the rete mirabile. Three different specimens are shown as examples of grade 0 , no filling (A); grade I, incomplete filling (B); and grade II, complete filling of the proximal rete $(C)$. (Hematoxylin phloxine saffron stain; original magnification $\times 50$.)

\section{Summary of LEA injections}

\begin{tabular}{|c|c|c|c|c|c|}
\hline Group and Injection & Reflux & Extent & Density & $\begin{array}{c}\text { Technical } \\
\text { Complications }\end{array}$ & LEA Used \\
\hline \multicolumn{6}{|l|}{$\begin{array}{l}\text { Group A: dual-lumen balloon } \\
\text { catheter }\end{array}$} \\
\hline $1^{\mathrm{a}}$ & 2 & 3 & 2 & No & Onyx 18 \\
\hline $2^{\mathrm{a}}$ & 1 & 4 & 2 & No & Onyx 18 \\
\hline $3^{a}$ & 1 & 4 & 2 & No & PHIL \\
\hline $4^{\mathrm{a}}$ & 2 & 3 & 1 & No & PHIL \\
\hline $5^{a}$ & 1 & 4 & 2 & No & PHIL \\
\hline 6 & 3 & 3 & 1 & No & PHIL \\
\hline $7^{\mathrm{a}}$ & 2 & 3 & 2 & No & Onyx 18 \\
\hline 8 & 3 & 4 & 2 & No & Onyx 18 \\
\hline \multicolumn{6}{|c|}{$\begin{array}{l}\text { Group B: double-microcatheter } \\
\text { technique }\end{array}$} \\
\hline 9 & 3 & 2 & 2 & No & Onyx 18 \\
\hline 10 & 3 & 3 & 1 & Yes & Onyx 18 \\
\hline 11 & 3 & 4 & 2 & No & Onyx 18 \\
\hline 12 & 3 & 3 & 2 & Yes & Onyx 18 \\
\hline \multicolumn{6}{|c|}{$\begin{array}{l}\text { Group C: single-microcatheter } \\
\text { (controls) }\end{array}$} \\
\hline 13 & 3 & 1 & 1 & No & Onyx 18 \\
\hline 14 & 3 & 3 & 1 & Yes & PHIL \\
\hline 15 & 3 & 3 & 1 & No & PHIL \\
\hline 16 & 3 & 1 & 0 & No & Onyx 18 \\
\hline $17^{a}$ & 2 & 4 & 1 & Yes & PHIL \\
\hline 18 & 3 & 1 & 1 & No & Onyx 18 \\
\hline
\end{tabular}

${ }^{a}$ Denotes a successful injection, defined as an injection with a grade $>2$ for extent of infiltration without excessive reflux (reflux score of $<3$ ).

using a 3-point scale as follows: grade I, acceptable reflux (limited to the distal two-thirds of the ascending pharyngeal artery); grade II, maximal permissible reflux (up to the proximal one-third of the ascending pharyngeal artery); and grade III, reflux into any artery other than the ascending pharyngeal, which was considered unacceptable.

The density of LEAs within the proximal rete mirabile was scored after microscopic examination by the same raters by using a 3-point scale, adjudicated as follows: 0 , no filling; 1, partial filling; and 2, total filling of the proximal rete. A successful liquid embolic injection was defined as an injection that reached an extent grade of $>2$ without excessive reflux (a reflux score of $<3$ ). In case of discrepancies between microscopic examination and macrophotographs, a final verdict was established through a consensus session. At the end of each injection and for each microcatheter, the amount of traction required to retrieve the catheter was assessed. Inflated balloon-catheters were deflated under fluoroscopic control. The distal tips of all catheters, including the $0.5-\mathrm{mm}$ distal catheter tip of the Scepter balloon, were examined and photographed by using a stereomicroscope, looking for adherent embolic material or fragments.

\section{Technical Complications}

\section{Statistics}

Groups were compared by exact logistic regression analyses, by using .05 as the threshold for statistical significance. Interobserver agreement in scoring the extension of rete embolization was assessed by using a weighted $\kappa$ with a $95 \%$ confidence interval. ${ }^{17}$ The $\kappa$ values were interpreted according to Alt$\operatorname{man}^{18}$ : $\leq 0.20$, poor agreement; $0.21-$ 0.40 , fair agreement; $0.41-0.60$, moderate agreement; 0.61-0.80, good agreement; and $0.81-1.00$, very good agreement.

\section{RESULTS}

Results are summarized in the Table and illustrated in Figs 1 and 2.

The extent of rete embolization was $>2$ in 8/8 dual-lumen balloon animals (group A), 3/4 double-microcatheter animals (group B), and 3/6 single-microcatheter animals (group C) $(P=$ .073 ). There was good (raters 1 versus 3 and 2 versus $3 ; 70 \%$ concordance; $\kappa=$ 0.8 [range, $0.625-0.975$ ]) to very good (rater 1 versus 2; 100\% concordance; $\kappa=1$ ) agreement in scoring extension of embolization between observers. Excessive (or grade 3 ) reflux occurred in $4 / 4$ group $B$ and 5/6 group $C$ but only in $2 / 8$ group A animals $(P=.029)$. The density of LEA differed among groups $(P=.014)$, with the rete mirabile of the group A animals found to be more densely filled than that of controls $(P=$ .019).

In 2 injections of the control group, the microcatheter tip was trapped within the LEA plug. In 2 animals treated by using the 2-microcatheter technique (group B), serious complications occurred, with extensive reflux (of LEA in 1 case and $n$-BCA in the other), leading to inadvertent embolization of branches. On 2 occasions during LEA embolization, the inflated balloon moved proximally by approximately $1 \mathrm{~cm}$ when the injection pressure was increased to more completely fill the contralateral rete. The retrograde balloon movement was controlled by gently further inflating the balloon. These cases were considered minor problems. Macroscopic inspection of the microcatheters did not reveal any material on balloon surfaces or catheter tips, but there were a few microscopic particles of the LEA mixture in 2 cases. There was no instance of spontaneous balloon deflation, and balloons remained intact in all cases.

The frequency of injections judged to be "successful" differed among groups $(P=.004)$, with more successful injections in the dual-lumen-balloon group (group A) animals compared with controls (group C, $P=.019$ ). 


\section{DISCUSSION}

The main result of this investigation is that dual-lumen balloon catheters can be used to allow LEA to more readily reach the contralateral rete without excessive reflux compared with singlelumen catheter or 2-microcatheter techniques. The balloon remains intact and can be deflated and retrieved easily at the end of the injection.

There are several important steps to successful injection of LEAs, which have been summarized by Weber et al. ${ }^{19}$ Most operators used a "push and plug" technique, ${ }^{20}$ in which after establishing a small proximal plug of LEA, the operator resumes the injection, assessing under close fluoroscopic guidance whether the LEA is moving antegrade to fill the nidus or refluxing proximally. The operator halted the injection for at least 2 reasons: 1 ) Too much reflux threatened to fill an unwanted more proximal blood vessel, potentially leading to an ischemic insult; or 2) reflux increased the length of catheter exposed to the solidifying LEA, which can increase the risk of trapping the catheter. In this work, the use of a dual-lumen balloon-mounted catheter effectively mitigated these problems.

The dual-lumen balloon-mounted catheters studied in this work have already been used in human applications, including brain arteriovenous malformations and dural arteriovenous fistulas, leading to several early, enthusiastic clinical reports. ${ }^{5,20,21}$ One potential advantage of the balloon catheter is the ability to inject LEA, perhaps more safely, in the presence of a high-flow shunt. $9,21,22$

To avoid complications, one must pay close attention, nonetheless, to the radiopaque LEA as it progresses from the microcatheter; retrograde flow can still occur despite an inflated balloon, and antegrade flow of LEA can still fill potentially dangerous anastomotic vessels.

Finally, the dual-lumen balloon catheter cannot be navigated as far distal in the vasculature as a simple microcatheter because it has a larger outer diameter. If an optimal position cannot be reached, a more proximal position from which to perform the injection risks filling of normal vessels "en route" to the nidus, with potential complications.

\section{Limitations}

One important limitation of this study is the small number of animals; in addition, groups were of unequal sizes, both affecting the power of statistical analyses. Although the rete mirabile is a plexiform vascular structure, it does not contain arteriovenous shunts. The surgical creation of an arteriovenous fistula can make this model more similar to an AVM. ${ }^{23,24}$ It is unclear whether results would have differed if this more complex model had been used. The persistence of variable antegrade flow from the contralateral ascending pharyngeal artery may have affected the extension of the embolic agent when injected in the contralateral rete. In our hands, the surgical creation of a shunt may also introduce variable flow patterns in the model, from one animal to another. In addition, the shunt may decrease with time in some animals, making timing of experiments critical.

We have used a simple but subjective grading system, rather than a previously published, more objective method, to quantify the extension of LEA embolization. ${ }^{25}$ Two different LEAs were used during these experiments, but we think that this had no important effect on results. The use of the prototype agent PHIL was balanced among groups ( 4 of 8 injections for group A; 3 of 6 injections for group C). The rete is also considerably smaller than many AVMs encountered in patients, and complete occlusion in this experimental setting is more readily achievable. Injection times were also shorter than many injections in clinical embolizations, which can reach 45-60 minutes or more. This shorter period may not have allowed as much LEA hardening as can sometimes be required in the clinical setting; therefore, we may have underestimated the traction forces required for microcatheter removal. Other factors important for successful AVM embolization are not evaluable by using this model, including navigability of the balloon-catheter through tortuous vascular structures. The risks of specific complications such as vascular rupture arising from inflating balloons in dysplastic intracranial AVM feeders could not be assessed. We have not tested, in this model, other LEAs, such as $n$-BCA mixed with Lipiodol (Guerbet, Roissy, France), which cannot be used with the Scepter; the use of these agents may lead to balloon rupture. ${ }^{26}$

\section{CONCLUSIONS}

Balloon catheters may improve the control of LEA embolization in an animal model of vascular malformation.

\section{ACKNOWLEDGMENTS}

This publication was made possible in part by an International Fellowship award to Jean-Christophe Gentric by the Société Française de Radiologie.

Disclosures: Jean Raymond—RELATED: Grant: MicroVention provided unrestricted funding for this project.* *Money paid to the institution.

\section{REFERENCES}

1. Saatci I, Geyik S, Yavuz K, et al. Endovascular treatment of brain arteriovenous malformations with prolonged intranidal Onyx injection technique: long-term results in 350 consecutive patients with completed endovascular treatment course. J Neurosurg 2011;115:78-88

2. Pierot L, Cognard C, Herbreteau D, et al. Endovascular treatment of brain arteriovenous malformations using a liquid embolic agent: results of a prospective, multicentre study (BRAVO). Eur Radiol 2013;23:2838-45

3. van Rooij WJ, Jacobs S, Sluzewski M, et al. Curative embolization of brain arteriovenous malformations with Onyx: patient selection, embolization technique, and results. AJNR Am J Neuroradiol 2012;33:1299-304

4. Mounayer C, Hammami N, Piotin M, et al. Nidal embolization of brain arteriovenous malformations using Onyx in 94 patients. AJNR Am J Neuroradiol 2007;28:518-523

5. Jagadeesan BD, Grigoryan M, Hassan AE, et al. Endovascular balloon-assisted embolization of intracranial and cervical arteriovenous malformations using dual-lumen coaxial balloon microcatheters and Onyx: initial experience. Neurosurgery 2013;73(2 Suppl Operative):ons238-43

6. ev3 Onyx Liquid Embolic System: Safety Communication-Risk of Catheter Entrapment. Posted June 29, 2012. http://www.fda. gov/Safety/MedWatch/SafetyInformation/SafetyAlertsforHuman MedicalProducts/ucm310199.htm. Accessed June 19, 2014

7. Shi ZS, Loh Y, Duckwiler GR, et al. Balloon-assisted transarterial embolization of intracranial dural arteriovenous fistulas. J Neurosurg 2009;110:921-28 
8. Newman CB, Hu YC, McDougall CG, et al. Balloon-assisted Onyx embolization of cerebral single-channel pial arteriovenous fistulas. J Neurosurg Pediatr 2011;7:637-42

9. Andreou A, Ioannidis I, Nasis N. Transarterial balloon-assisted glue embolization of high-flow arteriovenous fistulas. Neuroradiology 2008;50:267-72

10. Spiotta AM, James RF, Lowe SR, et al. Balloon-augmented Onyx embolization of cerebral arteriovenous malformations using a dual-lumen balloon: a multicenter experience. J Neurointerv Surg 2014 Aug 12. [Epub ahead of print]

11. Raymond J, Salazkin I, Gevry G, et al. Interventional neuroradiology: the role of experimental models in scientific progress. AJNR Am J Neuroradiol 2007;28:401-05

12. Murayama Y, Viñuela F, Ulhoa A, et al. Nonadhesive liquid embolic agent for cerebral arteriovenous malformations: preliminary histopathological studies in swine rete mirabile. Neurosurgery 1998;43:1164-175

13. Natarajan SK, Born D, Ghodke B, et al. Histopathological changes in brain arteriovenous malformations after embolization using Onyx or N-butyl cyanoacrylate: laboratory investigation. J Neurosurg 2009;111:105-13

14. Spiotta AM, Sivapatham T, Teng Q, et al. Balloon-augmented carotid artery sacrifice with Onyx: a proof of concept study in a swine model. J Neurointerv Surg 2011;3:390-94

15. Amiridze NS, Darwish R, Griffith GM, et al. Treatment of arteriovenous malformations with hydrocoils in a swine model. Interv Neuroradiol 2008;14:165-71

16. Levrier $\mathrm{O}$, Mekkaoui $\mathrm{C}$, Rolland $\mathrm{PH}$, et al. Efficacy and low vascular toxicity of embolization with radical versus anionic polymerization of n-butyl-2-cyanoacrylate (NBCA): an experimental study in the swine. J Neuroradiol 2003;30:95-102

17. Fleiss JL, Levin B, Paik MC. Statistical Methods for Rates and Proportions. 3rd ed. New York: Wiley; 2003
18. Altman DG. Practical Statistics for Medical Research. London: Chapman and Hall; 1990

19. Weber W, Kis B, Siekmann R, et al. Endovascular treatment of intracranial arteriovenous malformations with Onyx: technical aspects. AJNR Am J Neuroradiol 2007;28:371-77

20. Paramasivam S, Niimi Y, Fifi J, et al. Onyx embolization using duallumen balloon catheter: initial experience and technical note. J Neuroradiol 2013;40:294-302

21. Orozco LD, Luzardo GD, Buciuc RF. Transarterial balloon assisted Onyx embolization of pericallosal arteriovenous malformations. J Neurointerv Surg 2013;5:e18

22. Jagadeesan BD, Mortazavi S, Hunter DW, et al. Endovascular balloon-assisted embolization of high-flow peripheral vascular lesions using dual-lumen coaxial balloon microcatheter and Onyx: initial experience. J Vasc Interv Radiol 2014;25:587-92

23. Siekmann R, Wakhloo AK, Lieber BB, et al. Modification of a previously described arteriovenous malformation model in the swine: endovascular and combined surgical/endovascular construction and hemodynamics. AJNR Am J Neuroradiol 2000;21:1722-25

24. Massoud TF, Ji C, Viñuela F, et al. An experimental arteriovenous malformation model in swine: anatomic basis and construction technique. AJNR Am J Neuroradiol 1994;15:1537-45

25. Lieber BB, Wakhloo AK, Siekmann R, et al. Acute and chronic swine rete arteriovenous malformation models: effect of ethiodol and glacial acetic acid on penetration, dispersion, and injection force of N-butyl 2-cyanoacrylate. AJNR Am J Neuroradiol 2005;26:1707-14

26. Park S, Hwang SM, Lim OK, et al. Compliant neurovascular balloon catheters may not be compatible with liquid embolic materials: intraprocedural rupture of the protecting balloon during tumor embolization using n-butyl cyanoacrylate and lipiodol mixture. J Neurointerv Surg 2014 Aug 27. [Epub ahead of print] 